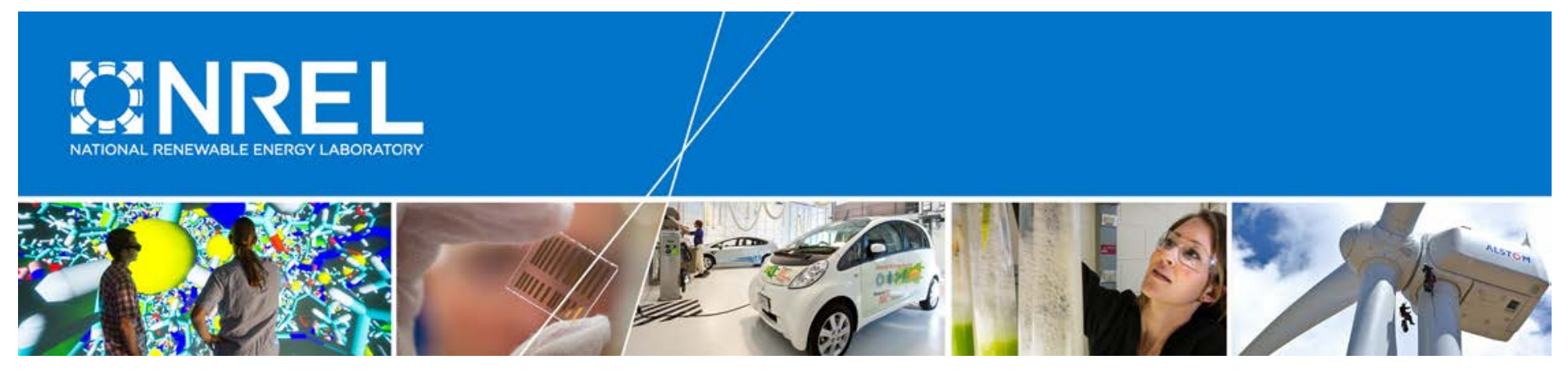

\title{
An Advanced Actuator Line Method for Wind Energy Applications and Beyond
}

\section{Preprint}

Matthew Churchfield and Scott Schreck National Renewable Energy Laboratory

Luis A. Martínez-Tossas and Charles Meneveau Johns Hopkins University

Philippe R. Spalart Boeing Commercial Airplanes

Presented at the American Institute of Aeronautics and Astronautics SciTech 2017 Grapevine, Texas January 9-13, 2017

NREL is a national laboratory of the U.S. Department of Energy Office of Energy Efficiency \& Renewable Energy Operated by the Alliance for Sustainable Energy, LLC

This report is available at no cost from the National Renewable Energy Laboratory (NREL) at www.nrel.gov/publications.

Conference Paper NREL/CP-5000-67611

March 2017 


\section{NOTICE}

The submitted manuscript has been offered by an employee of the Alliance for Sustainable Energy, LLC (Alliance), a contractor of the US Government under Contract No. DE-AC36-08GO28308. Accordingly, the US Government and Alliance retain a nonexclusive royalty-free license to publish or reproduce the published form of this contribution, or allow others to do so, for US Government purposes.

This report was prepared as an account of work sponsored by an agency of the United States government. Neither the United States government nor any agency thereof, nor any of their employees, makes any warranty, express or implied, or assumes any legal liability or responsibility for the accuracy, completeness, or usefulness of any information, apparatus, product, or process disclosed, or represents that its use would not infringe privately owned rights. Reference herein to any specific commercial product, process, or service by trade name, trademark, manufacturer, or otherwise does not necessarily constitute or imply its endorsement, recommendation, or favoring by the United States government or any agency thereof. The views and opinions of authors expressed herein do not necessarily state or reflect those of the United States government or any agency thereof.

This report is available at no cost from the National Renewable Energy Laboratory (NREL) at www.nrel.gov/publications.

Available electronically at SciTech Connect http:/www.osti.gov/scitech

Available for a processing fee to U.S. Department of Energy and its contractors, in paper, from:

U.S. Department of Energy

Office of Scientific and Technical Information

P.O. Box 62

Oak Ridge, TN 37831-0062

OSTI http://www.osti.gov

Phone: 865.576.8401

Fax: 865.576.5728

Email: reports@osti.gov

Available for sale to the public, in paper, from:

U.S. Department of Commerce

National Technical Information Service

5301 Shawnee Road

Alexandria, VA 22312

NTIS http://www.ntis.gov

Phone: 800.553 .6847 or 703.605 .6000

Fax: 703.605.6900

Email: orders@ntis.gov 


\title{
An Advanced Actuator Line Method for Wind Energy Applications and Beyond
}

\author{
Matthew J. Churchfield* and Scott Schreck ${ }^{\dagger}$ \\ National Renewable Energy Laboratory, Golden, Colorado 80401, USA \\ Luis A. Martínez-Tossas ${ }^{\ddagger}$ and Charles Meneveau ${ }^{\S}$ \\ Johns Hopkins University, Baltimore, Maryland 21218, USA \\ Philippe R. Spalart \\ Boeing Commercial Airplanes, Seattle, Washington 98124, USA
}

\begin{abstract}
The actuator line method to represent rotor aerodynamics within computational fluid dynamics has been in use for more than a decade. This method applies a body force to the flow field along rotating lines corresponding to the individual rotor blades and employs tabular airfoil data to compute the force distribution. The actuator line method is attractive because, compared to blade-resolved simulations, the required mesh is much simpler and the computational cost is lower. This work proposes a higher fidelity variant of the actuator line method meant to fill the space between current actuator line and blade-resolved simulations. It contains modifications in two key areas. The first is that of freestream velocity vector estimation along the line, which is necessary to compute the lift and drag along the line using tabular airfoil data. Most current methods rely on point sampling in which the location of sampling is ambiguous. Here we test a velocity sampling method that uses a properly weighted integral over space, removing this ambiguity. The second area of improvement is the function used to project the one-dimensional actuator line force onto the three-dimensional fluid mesh as a body force. We propose and test a projection function that spreads the force over a region that looks something like a real blade with the hope that it will produce the blade local and near wake flow features with more accuracy and higher fidelity. Our goal is that with these two improvements, not only will the flow field predictions be enhanced, but also the spanwise loading will be made more accurate. We refer to this combination of improvements as the advanced actuator line method. We apply these improvements to two different wind turbine cases. Although there is a strong wind energy motivation in our work, these advanced actuator line ideas can also be used in other applications, such as helicopter rotors.
\end{abstract}

\section{Introduction}

$\mathrm{T}^{\mathrm{N}}$ predicting the flow through wind plants or about helicopters using computational fluid dynamics (CFD), often the rotor aerodynamics are modeled using an actuator method such as the actuator line or disk. The focus of this work is on an improved, higher fidelity, actuator-line implementation, and our main application is wind energy.

The actuator line model represents each rotor blade as a line of radially varying force. The radially varying velocity is estimated along the line in some way, from which velocity magnitude and angle of attack are computed. Those values are used as inputs to appropriate airfoil tables that return lift and drag along the line. Commonly, various airfoil tables are used along the blade radius because the blade airfoil type

*Senior Engineer, National Wind Technology Center, matt.churchfield@nrel.gov, AIAA Member.

$\dagger$ Principal Engineer, National Wind Technology Center, AIAA Member

$\ddagger$ Ph.D. Student, Department of Mechanical Engineering, AIAA Member

$\S$ Professor, Department of Mechanical Engineering, AIAA Member

ฯSenior Technical Fellow, AIAA Member 
usually changes with radius, as does the operating Reynolds number. The lift and drag distribution along each actuator line is then projected onto the CFD mesh using some sort of projection function. The actuator lines rotate in space like the rotor blades do. They can be coupled with a rotor inertia and control system model so that the actuator line rotor dynamically responds to changing conditions. If coupled to a structural dynamics solver, the actuator lines can be made to deflect like real elastic rotor blades. The actuator line model, because of its discrete blade-like forces, as opposed to the actuator disk model that applies force over the entire rotor disk at once, creates gross rotor flow features like root and tip vortices and blade-local axial induction zones. On the other hand, since the surface of each blade is not modeled, the actuator line model does not replicate finer flow features including the blade boundary layer and separation at high angle of attack. Because the blade boundary layer is not resolved, the actuator line model is less computationally expensive than blade-resolved CFD in terms of both mesh resolution requirements and time step size. For these reasons, the actuator line model has become a popular choice in wind plant CFD, particularly largeeddy simulations (LES) with multiple turbines imbedded within the atmospheric boundary layer.

The first researchers to document their application of the actuator line concept to wind energy are Sørensen and Shen. ${ }^{1}$ Their basic actuator line concept has been applied by many other wind energy researchers, including Mikkelsen et al., ${ }^{2}$ who use actuator lines to study wake interactions in a row of turbines. Troldborg's Ph.D. thesis ${ }^{3}$ is completely devoted to the application and better understanding of the actuator line. Troldborg et al. ${ }^{4}$ show differences in computed wakes produced by actuator disks, actuator lines, and fully resolved rotors. Martínez-Tossas et al. ${ }^{5}$ study the effect of various actuator line parameters on the predicted wakes and power and compare actuator line results with those of a wind tunnel experiment. Churchfield et al. use the actuator line to study the impact of atmospheric stability on wind turbine power and mechanical loads, ${ }^{6}$ to simulate full wind plants, ${ }^{7,8}$ and to simulate various configurations of an array of tidal turbines. ${ }^{9} \mathrm{Lu}$ et al. ${ }^{10}$ and Bhaganagar and Debnath ${ }^{11}$ simulate wind turbines in stable atmospheric conditions using the actuator line model. Jha et al. ${ }^{12}$ and Crawford and Shives ${ }^{13}$ suggest improvements to the actuator line to better predict tip loads. Fleming et al. ${ }^{14,15}$ use the actuator line model to study wake-redirection through yaw misalignment as a wind-plant control strategy. This list surely is not exhaustive and it highlights the popularity of the actuator line method in wind energy CFD. The actuator line concept is not unique to wind energy; it also is used successfully in the helicopter and naval communites. An interesting application is by Forsythe et al. ${ }^{16}$ in the CFD simulation of a helicopter landing on a ship deck. Equally interesting is Jones and Paterson's ${ }^{17}$ computations of the flow around a submarine using actuator line propellers.

Two key steps to the actuator line method are: i) the sampling of the freestream velocity vector along the line for use by the airfoil lift and drag tables, and ii) the way in which the line force is projected onto the CFD mesh as a body-force field. Item i) is ambiguous. Airfoil tables give coefficients of lift and drag as a function of angle of attack, where the angle of attack is defined as the angle between the airfoil chord line and the freestream flow angle. Then converting from coefficients of lift and drag to absolute lift and drag requires the freestream velocity magnitude. The key word in both steps is freestream. That means the velocity sampling should be done in a way such that the blade-local flow effects (e.g., blade-circulation-induced upwash and downwash) are not seen, but the gross deceleration of the flow in the rotor axial induction zone must be seen because that is the freestream to which the blades are subject. In Sørensen and Shen's original actuator line paper, ${ }^{1}$ they state that the velocity sampling must be done, but do not state how. For simple actuator line force projection functions, such as the isotropic Gaussian used by Sørensen and Shen ${ }^{1}$ and most others, the bound vortex cross section is circular. Therefore, many actuator-line users sample the velocity directly along the actuator line, which is also the center of the bound vorticity where the blade-local flow effects are not seen. Martinez-Tossas et al. ${ }^{18}$ suggest that sampling at the center of the bound vortex should give the correct freestream velocity, although the velocity at the center of the bound vortex needs to be corrected in cases with high drag. However, if a more elaborate line-force projection function is used, sampling along the actuator line likely no longer avoids the blade-local flow effects. Others, such as Mittal et al., ${ }^{19}$ have experimented with sampling some distance upstream of the actuator line in an attempt to avoid sampling blade-local flow effects, but the solution is sensitive to the sampling location, which can be seen as a tunable parameter, and there is no general consensus on the proper sampling location. Also, upstream sampling in turbulent flow means the blade forces will react to turbulent structures before the actuator line actually encounters them. Mittal et al. ${ }^{19}$ also tried sampling at multiple locations to obtain an average velocity.

Item ii), the actuator-line-force projection method, is full of opportunity. The method that most researchers use, which follows Sørensen and Shen, ${ }^{1}$ projects the line force into a body-force field using a 
three-dimensional Gaussian function that is isotropic in width and fixed in width along the blade span. We have interpreted their method as first dividing up each actuator line into a series of small actuator elements, each of which has a lift and drag force at its center point. At each CFD mesh cell within a region surrounding the actuator line, a body force is assigned that has units of force per unit volume. The body force at each mesh cell is the summation of the projected actuator element forces. Because the projection function is Gaussian, the actuator element nearest a particular CFD mesh cell has the most influence on that cell. The volume-integrated body-force field must equal the total actuator line force. With the isotropic Gaussian projection, the resultant body-force field around an actuator line appears like a cylindrical cloud surrounding the line. Sørensen and Shen ${ }^{1}$ state that the reason for applying the body force is to smoothly distribute the actuator line force from a single actuator element onto more than one CFD mesh point or volume, presumably to maintain a solution free of spurious oscillations in the actuator line vicinity. Although we agree with that reasoning, we feel that the line-force projection function also has a physical relevance. On a real wind turbine blade, airplane wing, or helicopter rotor blade, the force is spread out over the entire surface, so the body force also can be spread out to mimic this physical reality.

Although currently used actuator line methods, which are nearly the same as or close derivatives of Sørensen and Shen's original method, ${ }^{1}$ work reasonably well, there are some issues that could be improved. For example, it is well known that the tip loads are not well predicted, as is clearly shown by Jha et al. ${ }^{12}$ Shen, Sørensen, and Mikkelsen ${ }^{20}$ remedy this through the use of a tip and root-loss correction, but we feel that because the actuator line has discrete tips, there should not be the need for a tip- and root-loss correction. Shives and Crawford ${ }^{13}$ attempt to address this through a three-dimensional Gaussian that is isotropically wide, but that varies in width along the blade radius as a linear function of local chord length. Jha et al. ${ }^{12}$ build upon the work of Shives and Crawford, ${ }^{13}$ but they do not scale the Gaussian width directly with chord, but with an equivalent elliptical planform chord. Also, Martínez-Tossas et al. ${ }^{5}$ show that that actuator line has trouble accurately simultaneously matching experimentally measured power and thrust. The power can be well predicted, but then the thrust is too low. This may be a result of the fact that an isotropic Gaussian function projects the line force beyond the tips, in effect making the turbine blade appear more efficient than it really is. Related is the fact that because the body force exponentially dies off away from the tips rather than discretely ending at the tips, the tip vortices are rather diffuse and the wake shear layer is likely weaker than in reality. This is especially important for helicopters in which the blades interact with the tip vortices of other blades. Last, the loads predicted along the rotor are highly dependent on the width of the line force projection function, as shown by Martínez-Tossas et al. ${ }^{5}$ In fact, if a very large projection width is chosen, the actuator line model will recover an aerodynamic power in excess of the Betz limit, and if too small a value is chosen, the predicted power will be well below measurements or blade-element momentum calculations. We have found that when using the isotropic Gaussian projection function, the function width should be about 0.035 times the rotor diameter. This is simply a rule of thumb and seems to hold fairly well for high aspect ratio rotor blades, but breaks down on smaller aspect ratio blades, such as those used in marine and hydrokinetic turbines. The value varies somewhat for different rotors and grid resolutions, so it is not a hard rule. Martínez-Tossas et al. ${ }^{18}$ show that an optimal force projection width can be determined semianalytically by minimizing the difference between the velocity fields in inviscid flow subjected to a Gaussian body force and potential flow solution over a generic airfoil. They obtain optimal widths on the order of $20-40 \%$ of the chord length, although these insights have not been well tested in LES. With this variety of conclusions, more exploration is clearly needed.

The objective of this work is to present a more robust and higher fidelity version of the actuator line method that improves upon the currently used method. Our overall goal is to move toward an actuator line method that more accurately predicts both blade loading and the resultant near-field and far-field wakes, that more physically realistically represents the flow around a real blade, and that has as few tunable or ambiguous parameters as possible. We envision this actuator line occupying a fidelity space somewhere between the current actuator line and blade-resolved simulations. Increased fidelity incurs more computational expense and complexity, but not at the level of blade-resolved simulations. To make progress toward our goal, we are developing an actuator line force projection function that has more physical relevance and is able to more sharply capture the root and tip vortices and blade wake. We are also developing an unambiguous method for sampling the velocity along the blade. From this point forward, the developments outlined here are collectively referred to as the "advanced actuator line (AAL)" and the currently used method as the "actuator line (AL)." We will test the new developments on both subscale and utility-scale wind turbine cases. We titled this paper "An Advanced Actuator Line Method for Wind Energy Applications and Beyond" 
because we will apply and test the method for wind turbines, but there is no reason it cannot be used for other applications such as helicopters or propellers.

\section{Method}

In this section, we outline how the AAL differs from the AL, and how we test the AAL. All work has been performed using a solver built from the OpenFOAM CFD toolbox. ${ }^{21}$ The solver is incompressible, implicit, and second-order accurate in space and time. Typically, we use linear interpolation of quantities to cell faces, which is equivalent to second-order central differencing on unstretched meshes, but in certain regions of the flow, especially when using nonturbulent inflow, a very small amount of upwinding needs to be added to maintain stability. Discretization in time is typically Crank-Nicolson. The governing equations are solved sequentially using the pressure implicit splitting operation (PISO). ${ }^{22,23}$ All variables are located at cell centers and Rhie-Chow ${ }^{24}$ interpolation is used to avoid velocity-pressure decoupling. Our work completely focuses on LES, but there is no reason the AAL could not be used with the unsteady Reynolds-averaged Navier-Stokes (RANS) equations. We use a one-equation subgrid-scale turbulent kinetic energy model to close the equations.

\section{II.A. Body Force Projection}

The purpose of the actuator line force to body-force projection function $g$ is simply to project the lift and drag force computed along the one-dimensional line, in our case at closely-spaced discrete actuator line element points, onto the three-dimensional CFD mesh as a body force. The function $g$ integrates to one. At the simplest, one could identify the CFD cells in which the actuator line passes and apply the line force to only those cells. However, such an abrupt force not only creates spurious numerical oscillations that pollute the flow field, but also may not be physically realistic since the lift and drag generated by a lifting body is not concentrated in a small region but rather spread over the body. In this section, we present the standard $\mathrm{AL}$ and the proposed AAL body-force projection functions. Figures 1 and 2 illustrate the differences in the approaches.

\section{II.A.1. AL Isotropic Gaussian Projection}

In the actuator line method outlined by Sørensen and Shen, ${ }^{1}$ the projection function is a three-dimensional Gaussian that is isotropic in width, and does not change width along the blade span. The function is given by

$$
g_{A L}(x, y, z)=\frac{1}{\epsilon^{3} \pi^{3 / 2}} \exp \left(-\frac{\left(x-x_{0}\right)^{2}+\left(y-y_{0}\right)^{2}+\left(z-z_{0}\right)^{2}}{\epsilon^{2}}\right),
$$

where $\epsilon$ is the Gaussian width; $x, y$, and $z$ are the three coordinate directions; and $x_{0}, y_{0}$, and $z_{0}$ are the location about which the Gaussian is being applied, which in our case is the actuator line element control point. Then the body force surrounding the control point is simply $\vec{f}(x, y, z)=g_{A L}(x, y, z) \vec{F}$, where $\vec{F}$ is the actuator line element force. This is repeated at each actuator element point. The Gaussian distributions at adjacent control points overlap each other so multiple control points can contribute body force to a single CFD mesh cell, and the effect of each control point at that particular cell are summed. The result is a cylindrical "tube" of force surrounding the actuator line. This cylindrical tube is well depicted in the left column of Fig. 1, which shows isosurfaces of the projection function $g_{A L}$ as applied to the NREL Phase VI rotor. ${ }^{25}$ Figure 2 (a) shows isosurfaces of the resultant in-plane (torque-producing) component of the body force on this rotor.

Martínez-Tossas et al. ${ }^{5}$ show that the predicted power, which is a direct indicator of predicted blade loads, is very sensitive to the isotropic Gaussian width, $\epsilon$. Some guidance is given in the literature concerning how to choose $\epsilon$. Troldborg ${ }^{3}$ states that $\epsilon$ should be at least twice the local grid cell length, a constraint that reduces spurious flow field oscillations to acceptable levels by adequately resolving the projection function. Martinez-Tossas et al. ${ }^{18}$ suggest using $\epsilon$ in the range of 0.2 times the chord length to minimize the error between the predicted actuator-line flow field and the potential flow solution for flat plate or Joukowski airfoils. The first author's rule of thumb based on correct power prediction is that for large utility-scale rotors with large aspect ratios, $\epsilon$ should be in the neighborhood of 0.035 times the rotor diameter. Again, this value is grid-resolution and rotor-geometry dependent, so it is not a hard rule. Jha et al. ${ }^{12}$ show that 


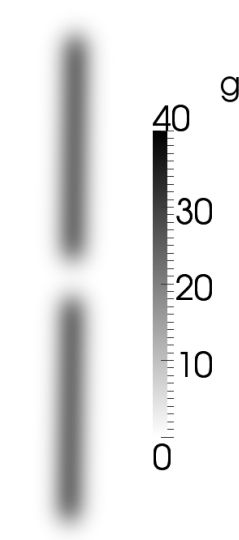

(a)

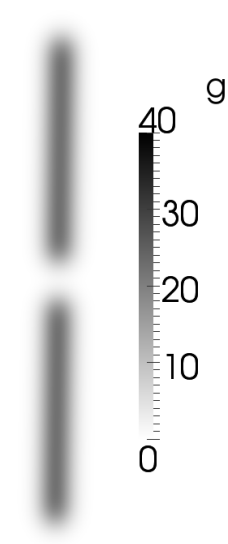

(c)

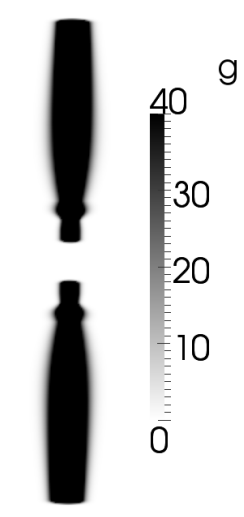

(b)

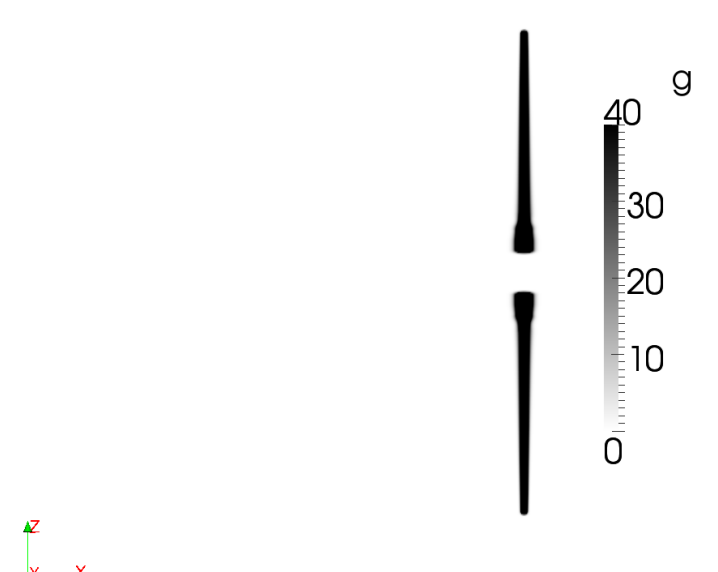

(d)

Figure 1. Contours of the body-force projection function as viewed from upstream (top row) and from the side (bottom row) for the isotropic Gaussian projection function AL (left column) and the nonisotropic Gaussian projection function AAL (right column). Rotor rotation is about the $x$-axis. 


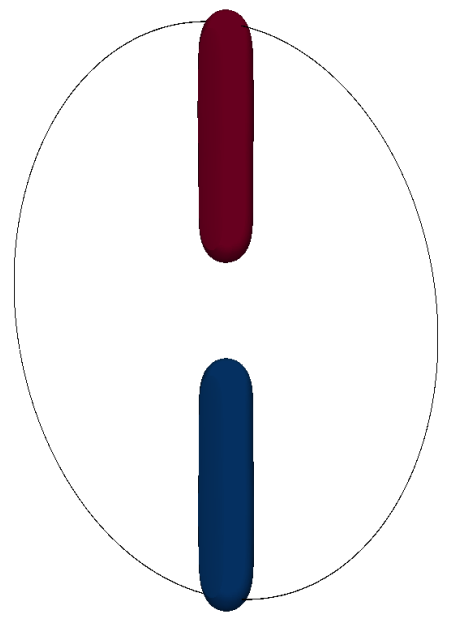

(a)

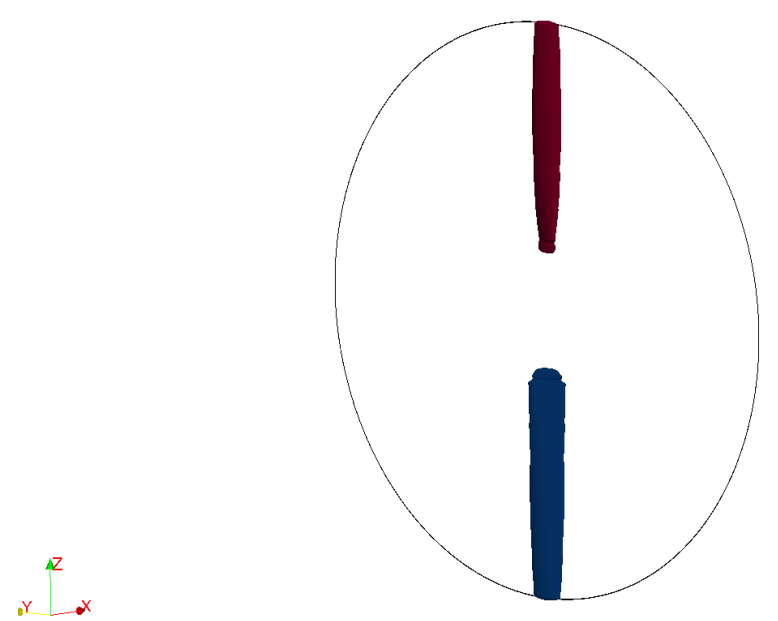

(b)

Figure 2. Isosurfaces of in-plane density-normalized body force (red is positive and blue is negative) for (a) the isotropic Gaussian projection function AL (contour level is $\pm 20 \mathrm{~m} / \mathrm{s}^{2}$ ) and (b) the nonisotropic Gaussian projection function AAL (contour level is $\pm 200 \mathrm{~m} / \mathrm{s}^{2}$ ). The view is from the front oblique, the black circle outlines the rotor extent, and rotor rotation is about the $x$-axis.

with the $g_{A L}$ function of Eq. (1), that is constant width along the span, the tip loads are overpredicted. Both Jha et al. ${ }^{12}$ and Shives and Crawford ${ }^{13}$ have proposed modifications in which the same Gaussian function is used, but the width $\epsilon$ varies along the blade span. The body force created by Eq. (1) also extends beyond the blade/actuator line tips artificially making the blade appear to the flow field to have a longer span than it really does. Although this method spreads the body force out over some region of the flow, and blade force is spread out over the blade, an isotropic Gaussian looks more like a cylinder than like a blade.

\section{II.A.2. AAL Nonisotropic Gaussian Projection}

We propose a variation on the isotropic Gaussian projection of Sørensen and Shen ${ }^{1}$ that makes the projection function, and hence the body force, look more like the force distribution of an airfoil. The new function is

$$
g_{A A L}\left(x_{c}, x_{t}, x_{r}\right)=\frac{1}{\epsilon_{c} \epsilon_{t} \epsilon_{r} \pi^{3 / 2}} \exp \left(-\frac{\left(x_{c}-x_{c, 0}\right)^{2}}{\epsilon_{c}^{2}}-\frac{\left(x_{t}-x_{t, 0}\right)^{2}}{\epsilon_{t}^{2}}-\frac{\left(x_{r}-x_{r, 0}\right)^{2}}{\epsilon_{r}^{2}}\right),
$$

where $x_{c}, x_{t}$, and $x_{r}$ are the coordinates in the chord-wise, thickness-wise, and radial directions, respectively. In real situations, these coordinate directions are a function of location on the blade span because the blade twists. The subscript "0" denotes the location about which the Gaussian is applied. The Gaussian widths in each of the three directions are $\epsilon_{c}, \epsilon_{t}$, and $\epsilon_{r}$. We relate these widths to the blade as follows: $\epsilon_{c}=a_{c} c$, $\epsilon_{t}=a_{t} t$, and $\epsilon_{r}=a_{r} \delta r$, where $c$ is the blade chord length, $t$ is the maximum thickness length, $\delta r$ is actuator element width, and $a_{c}, a_{t}$, and $a_{r}$ are dimensionless scalars. An isosurface of this projection function appears as an ellipsoid at a particular control point. If applied to all control points, the projection functions overlap, and an isosurface of the summation of the projection functions is longer in the chord direction than in the thickness direction, and follows the twist of the blade, which is well illustrated in the right side of Fig. 1, which shows $g_{A A L}$ applied to the NREL Phase VI wind turbine rotor. ${ }^{25}$ Figure 2 (b) shows isosurfaces of the resultant in-plane component of the body forces on this same rotor. Compared to using $g_{A L}$, the proposed $g_{A A L}$ function clearly distributes the forces in a way that resembles the actual blade shape and that well captures the blade tips. Further testing will allow us to see if there is are universal values of the tunable parameters $a_{c}, a_{t}$, and $a_{r}$ that recover accurate loads distributions over the blade span. Martínez-Tossas et al. ${ }^{18}$ have shown that for two-dimensional flow, $\epsilon_{c} \approx 0.4 c$ and $\epsilon_{t} \leq 0.2 c$ are optimal in terms of error between 
the predicted flow field and the potential flow solution for flat plate or Joukowski airfoils. It still remains to be seen what the implications of using such values are on predicted load distribution and power predictions. We will also show in the results section that the $g_{A A L}$ force distribution creates a less diffuse tip vortex with larger peak strength.

Because this version of the projection function is thin in the blade thickness direction, except at the blade root that is usually circular, higher grid resolution near the blades is necessary to well resolve the projection function and the resultant flow features. This can be seen as a tradeoff between computational cost and more realistic near blade and near wake flow. It also probably requires the actuator line to be divided into smaller actuator elements, which also adds to computational cost. For each actuator element, a search must be done over the cells in the vicinity of the blade to find the ones in which the line force will be appreciably projected.

\section{II.B. Velocity Sampling}

The purpose of sampling the freestream velocity vector along the actuator line is to compute angle of attack, which is input to the airfoil lift and drag tables, and to obtain velocity magnitude, which is used to dimensionalize the coefficients of lift and drag returned from the airfoil tables. Airfoil tables are often created by placing a wing of constant airfoil section in a wind tunnel, measuring its lift and drag as a function of a known freestream wind vector and wing pitch angle. Therefore, the freestream wind vector is measured far enough upstream of the wing such that the local flow effects induced by the wing are not present. For a wind turbine blade, that freestream wind vector is difficult to characterize. It is the wind vector in the blade rotation plane that contains the gross rotor axial induction, but it should not contain the blade-local induced velocity (e.g., the upwash just ahead of the blade).

\section{II.B.1. AL Pointwise Velocity Sampling}

With the standard AL method, the actuator line is split into a series of short actuator elements. Typically, we divide the line into 40-60 actuator elements. At the center of each actuator element is the control point. Velocity is sampled at each of these control points by linear interpolation from the surrounding CFD mesh. This method works well if the actuator line force projection function is symmetric in space, such as the isotropic Gaussian. The isotropic Gaussian creates a bound vortex around the actuator line that is circular in cross section (see Martínez-Tossas et al. ${ }^{18}$ for an explicit evaluation of such a vortex in inviscid flow). The actuator line lies at the center of the vortex where there is no vortex-induced velocity. Therefore, the point sampling along the line does not "feel" the effect of the local bound vorticity, as desired, but is still within the gross axial induction zone of the rotor. This method suffers from interpolation error, which becomes significant on meshes in which the bound vortex is marginally resolved, which is often the case. This method breaks down for line force projection functions in which there is no clear center of the bound vorticity. It is true that the velocity sampling location could be moved ahead of the actuator line and some researchers have tried that, including Mittal et al. ${ }^{19}$ Additionally, Shen et al. ${ }^{26}$ address the problem of how to determine angle of attack for a wind turbine blade, and their work is not specific to actuator line modeling. However, the predicted freestream velocity is sensitive to the sampling location, and that location becomes a tunable parameter, which is not desirable.

\section{II.B.2. AAL Integral Velocity Sampling}

To overcome the problem of an ambiguous velocity sampling location, Spalart ${ }^{27}$ devised a mathematically sound method of determining the freestream velocity using an integral of the velocity field weighted by the force projection function $g$. Forsythe et al. use this method for helicopters and describe it in the appendix of their paper. ${ }^{16}$

The derivation is discussed here for two-dimensional, steady, incompressible flow in the $(x, y)$ plane. In real applications, the flow is unsteady and three-dimensional. We assume the unsteadiness is at time scales long relative to the response time of the actuator-line-local flow to changes in body force. The variation in the flow along the blade span is weaker than in the other directions, so the two-dimensional assumption is valid.

As described in the previous section, the body force is projected using a function $g$ which integrates to one. The vector body force added to the momentum equation has a lift component orthogonal to the local 
velocity and a parasite drag component parallel to it. It is given by

$$
\vec{f}=-\rho \frac{c}{2} \hat{U}\left(C_{l} \vec{e}_{z} \times \vec{U}+C_{d p} \vec{U}\right) g(x, y),
$$

where $\vec{e}_{z}$ is the vector pointed along the wing or blade axis, $C_{l}$ and $C_{d p}$ are the coefficients of lift and parasite drag, $\vec{U}(x, y)$ is the local velocity of the fluid relative to the blade, and the scalar velocity $\hat{U}$ is to be determined. It can be the local velocity magnitude $|\vec{U}(x, y)|$, or be a single value attached to the entire airfoil (as $C_{l}$ and $C_{d p}$ are). Equation (3) is a proposed model form, which we now attempt to justify.

The steady incompressible momentum equation with body force can be written after standard vector manipulations (and of course $\vec{\omega}=\omega_{z} \vec{e}_{z}$ )

$$
\omega_{z} \vec{e}_{z} \times \vec{U}+\vec{\nabla}\left(\frac{p}{\rho}+\frac{|\vec{U}|^{2}}{2}\right)=\nu \nabla^{2} \vec{U}-\frac{c}{2} \hat{U}\left(C_{l} \vec{e}_{z} \times \vec{U}+C_{d p} \vec{U}\right) g(x, y)
$$

where $p$ is pressure, $\rho$ is density, and $\nu$ is viscosity. In real life, there will be a turbulence model and effective viscosity, but here we do not focus on viscous effects.

The vorticity transport equation is the curl of Eq. (4):

$$
\vec{U} \cdot \vec{\nabla} \omega_{z}=\nu \nabla^{2} \omega_{z}-\frac{c}{2}\left[C_{l} \vec{U} \cdot \vec{\nabla}(\hat{U} g)+C_{d p} \vec{e}_{z} \cdot \vec{\nabla} \times(\hat{U} g \vec{U})\right] .
$$

First, consider the basic situation for induced-drag purposes; the key difficulty in real applications is determining the "freestream" velocity to obtain the angle of attack to set $C_{l}$ and $C_{d p}$. We neglect viscosity, and parasite drag. Then there is only one term in the right-hand side of Eq. (5), and the vorticity is:

$$
\omega_{z}=-\frac{c C_{l}}{2} \hat{U} g(x, y)
$$

We get this by inverting the operator $\vec{U} \cdot \vec{\nabla}$, knowing that both fields, $\omega_{z}$ and $g$, are zero upstream. This equation shows how we are directly creating the vorticity, locally, with our body force, a convenient result. The form of $g$ is not limited to Gaussians as we propose above. One could shape the vorticity along the blade chord distribution, if desired. This result is consistent with the two-dimensional potential-flow situation, in which the support of the vorticity is the airfoil surface; here it is the support of the body force. The solution satisfies Bernoulli's equation everywhere, $p / \rho+|\vec{U}|^{2} / 2$ is constant. This is appropriate because the body force does no work since we defined it to be the cross product of the velocity vector with another vector in Eq. (3).

Using Eq. (6), the circulation around the airfoil is then

$$
\Gamma \equiv \iint \omega_{z} d x d y=-\frac{c C_{l}}{2} \iint \hat{U} g \mathrm{~d} x \mathrm{~d} y .
$$

which, using the Kutta theorem $L=\rho U \Gamma$, give a "Kutta lift"

$$
\vec{L}_{K}=\rho \frac{c C_{l}}{2}\left(\iint \hat{U} g \mathrm{~d} x \mathrm{~d} y\right) \vec{e}_{z} \times \vec{U}_{\infty}
$$

where $\vec{U}_{\infty}$ is the "freestream" velocity, obvious in a simple isolated airfoil, but not trivial at all in the real situation of a complex three-dimensional flow field. $\vec{U}_{\infty}$ :

There is another neat result from a few integrations by parts for an isolated vorticity region in a freestream

$$
\iint \omega_{z} \vec{U} \mathrm{~d} x \mathrm{~d} y=\Gamma \vec{U}_{\infty}
$$

That is, the average of the velocity vector, weighted by its own vorticity, is the freestream velocity. This is obvious for a distribution that is symmetric in $x$, say a Gaussian for the vorticity, but it is in fact general.

We now compare the Kutta lift from Eq. (8) with the negative of the integral of the body force in (4), which is the actual lift:

$$
\vec{L}_{b f}=\rho \frac{c C_{l}}{2} \vec{e}_{z} \times \iint \hat{U} \vec{U} g(x, y) \mathrm{d} x \mathrm{~d} y .
$$


Comparing Eq. (8) and Eq. (10), we recover the classical Kutta result if we define $\vec{U}_{\infty}$ as the average of $\vec{U}$ weighted by its vorticity (as in Eq. (9)) or, in other words, by $\hat{U} g$ (because of Eq. (6)):

$$
\vec{U}_{\infty} \equiv \frac{\iint \hat{U} g(x, y) \vec{U} \mathrm{~d} x \mathrm{~d} y}{\iint \hat{U} g(x, y) \mathrm{d} x \mathrm{~d} y}=\iint g(x, y) \vec{U} \mathrm{~d} x \mathrm{~d} y
$$

where the last equal sign is valid only if $\hat{U}$ has a single value.

We can now use airfoil tables, using $\vec{U}_{\infty}$ from Eq. (11) to give the angle of attack. Using such a unique velocity direction, attached to the whole airfoil, is superior to using the local velocity vector, which varies too much and depends on $g$.

The scalar velocity in the formula can be given by the local magnitude: $\hat{U} \equiv|\vec{U}|$, or by the magnitude of the global velocity: $\hat{U} \equiv\left|\vec{U}_{\infty}\right|$. The second solution is simpler and provides that the lift is exactly $1 / 2 \rho c C_{l}\left|\vec{U}_{\infty}\right| \vec{e}_{z} \times \vec{U}_{\infty}$, as per the definition.

To treat the drag, we take the dot product of the momentum equation given in Eq. (4) with $\vec{U}$, neglecting viscosity or turbulence model:

$$
\vec{U} \cdot \vec{\nabla}\left(\frac{p}{\rho}+\frac{|\vec{U}|^{2}}{2}\right)=-\frac{c C_{d p}}{2} \hat{U}|\vec{U}|^{2} g(x, y)
$$

We see the parasite-drag body force as the source term for a deficit in the Bernoulli total pressure, which will propagate into the wake. Equation (12) also gives the (negative) power of this force. The integral of the negative of the drag body force is

$$
\vec{D}_{b f}=\rho \frac{c C_{d p}}{2} \iint \vec{U} \hat{U} g(x, y) \mathrm{d} x \mathrm{~d} y=\rho \frac{c C_{d p}}{2} \hat{U} \vec{U}_{\infty},
$$

where the last equal sign is correct only if $\hat{U}$ is uniform and Eq. (11) applies exactly, which it does not when $C_{d p} \neq 0$. The integral in Eq. (9) also has a longer tail if there is drag. The definitions of $\vec{U}_{\infty}$ and $\hat{U}$ then again ensure that the drag is aligned with $\vec{U}_{\infty}$, as it should, with magnitude $D=c C_{d p} \rho\left|U_{\infty}\right|^{2} / 2$. This is to leading order in $C_{d p} / C_{l}$, but parasite drag is normally two orders of magnitude smaller than lift.

In summary, the key point of this derivation is the force-projection-function-weighted velocity integral of Eq. (11) that returns the "freestream" velocity vector. This derivation assumes a two-dimensional projection function $g$, and our hypothesis is that we can either use a three-dimensional projection function at each actuator point that decays quickly in the spanwise direction (i.e., a thin projection function that is much more dominant in the chord and thickness directions) or create an auxiliary cylindrical integration plane at each actuator point over which to integrate. The first option is the most convenient.

The region that can somewhat defeat this approach is at the tip, where variations along the span are not small. We hypothesize that the reaction of this system will be qualitatively correct: the spanload will adjust to gracefully fall to zero at the tip. However, we cannot expect the dependence on $g$ to be negligible. It will be a matter of wisely choosing $g$, and the grid. This will matter only if the grid is fine enough to resolve the tip vortex on the scale of the chord; if it is not, the method will give it the correct circulation, which is what matters most.

This method has similarities to that proposed by Mittal et al., ${ }^{19}$ but its mathematical foundation and integration using $g(x, y)$ as the weighting function is unique.

\section{II.C. Case To Be Studied}

Because one of the objectives of this work is to create an advanced actuator line method that more accurately predicts blade load distributions, we will simulate the NREL Phase VI wind tunnel test. ${ }^{25}$ In that experiment, a 10-m diameter rotor was placed in the NASA Ames 80-by-120 foot wind tunnel. Detailed blade spanwise load distributions were measured; we will compare to those measurements as well as to bladeelement-momentum theory. For this case, we match the conditions of test sequence $\mathrm{H}$ in which the wind tunnel flow speed at hub height is $7 \mathrm{~m} / \mathrm{s}$, the rotor speed is $71.9 \mathrm{rpm}$, and the blade pitch at the tip is $3^{\circ}$. We provide uniform, nonturbulent inflow that is similar to the very low turbulence level of the NASA Ames wind tunnel. 
Ultimately, we want to use the AAL for utility-scale wind plant simulations. Therefore, our other test case is the NREL 5-MW reference turbine, ${ }^{28}$ which is not an actual turbine but rather a fully characterized paper design of a utility-scale turbine used for many purposes, including model evaluation. No experimental data exists for this rotor, but we compare our results to blade-element-momentum calculations. For this case, the rotor is subject to uniform, nonturbulent, $8-\mathrm{m} / \mathrm{s}$ inflow wind, and the rotor speed is $9.1 \mathrm{rpm}$.

\section{Results}

\section{III.A. NREL Phase VI Cases: Subscale Wind Turbine in a Wind Tunnel}

In this section, we present the results of simulating the NREL Phase VI rotor. The mesh used for the computations is highly refined around the rotor disk to resolve the AAL body forces, which are more compact than with the AL method. The mesh in this region has cell lengths of $0.05 \mathrm{~m}$, which place roughly seven grid cells across the blade tip chord length. The mesh is also highly refined where the tip and root vortices will convect downstream. Away from the turbine and wake, the mesh is successively coarsened to conserve mesh points. The mesh is constructed by beginning with a base mesh of isotropic hexahedral elements. Sets of cells are selected for refinement and they are cut in half in each direction to achieve the desired resolution. We also used a coarser mesh that eliminated the $0.05-\mathrm{m}$ region such that the finest cells have $0.1-\mathrm{m}$ resolution, more typical of standard actuator line simulations. Unless otherwise noted, the results shown below are for the fine mesh. The fine mesh is roughly 50 million cells in size, which is large and a result of inefficient mesh refinement. A more efficient approach is to create a cylinder of rotating mesh about the rotor that rotates at the rotor speed. This mesh would only have fine resolution along the actuator lines, rather than throughout the entire cylinder, significantly reducing the number of mesh cells. The time step is $0.0025 \mathrm{~s}$ for both the coarse and fine cases. This limits the actuator line tip motion to pass through no more than one mesh cell per time step in the fine mesh case.

First, we give a qualitative overview of the resultant flow field in Figures 3-5. There we compare the AL to AAL. The images show a slice of instantaneous streamwise, or axial, velocity $18 \mathrm{~s}$ into the simulation. The cyan isosurfaces are of the second invariant of the velocity-gradient tensor, Q. The red isosurfaces are of the body-force projection function $g_{A L}$ or $g_{A A L}$. Qualitatively, the flow fields have some differences. Resolved-scale turbulence forms with less downstream distance in the AAL wake as compared to that of the AL wake. It is also important to point out that we used an actuator tower model ${ }^{8}$ to account for the drag created by the turbine tower. The tower wake is clearly visible in the velocity slices and it disturbs the helical tip vortices as they advect downstream. Figure 5 zooms in on the near-blade region. There, it is clear that the body-force projection function of the AAL is shaped similar to the Phase VI blade, whereas the body-force projection function of the AL is completely cylindrical. The tip vortex also is significantly more compact as it forms at the blade edge in the AAL case as compared to the AL case. In the AAL case, there is some spurious content that appears as thin Q isosurfaces adjacent to the main tip vortices near the rotor. Its origin is small-amplitude, spurious noise forming near the steep velocity gradients of the strong, compact tip vortices. We believe that additional resolution or the use of a more sophisticated advection scheme than the central differencing we use in this region would remedy this effect.

Next, we examine the actuator-line-local flow field. We sampled the velocity and body-force field upon annuli centered on the rotor hub with its axis aligned with the streamwise direction. Figure 6 shows a portion of the annulus with a radius of $75 \%$ of the blade radius. The annulus has been unrolled and flattened. It is cropped to show the flow field around only one of the blades because the other blade flow field looks nearly identical. The blade moves from left to right in these contour plots, and the wind-tunnel flow is from bottom to top. The first column of contours is of the axial velocity, the second column is of the tangential velocity in the blade moving reference frame, and the third column is the body-force magnitude normalized by density. The rows correspond to an AAL case, and two AL cases. The middle row AL case has a larger body-force projection width than the case shown in the lower row. The body-force contours for the AL body-force projection are round as expected because the Gaussian used is the same width in all directions. The AAL case body-force contours are elliptical and flow-aligned. The resultant flow field, which clearly shows the bound vorticity, responds to the different body-force shapes as expected. The contour lobes of tangential velocity in the AAL case are elongated in the blade-chord direction as compared to that of the AL case. The wake of the AAL actuator line is also more concentrated than in the AL case because the drag force is concentrated in the blade thickness direction. There are oscillations in tangential flow velocity about the AAL wake that we suspect may be eliminated by shaping the body force to follow the curved 


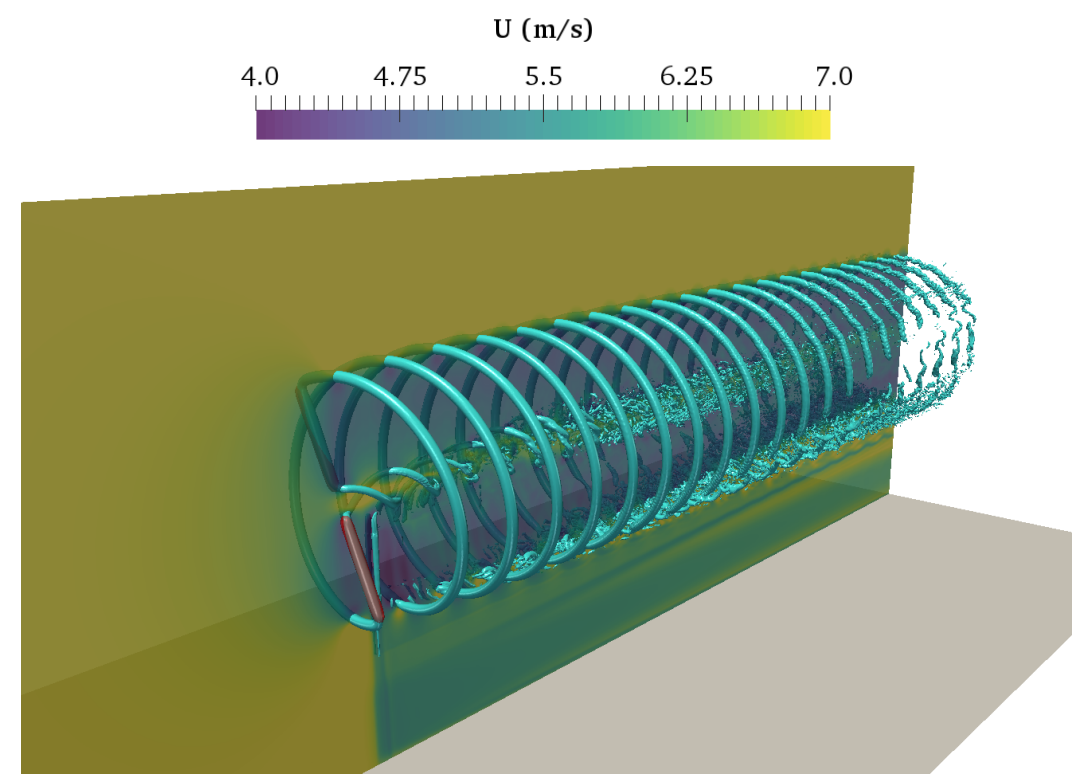

(a) AL, $\epsilon=0.235 \mathrm{~m}$, point velocity sampling

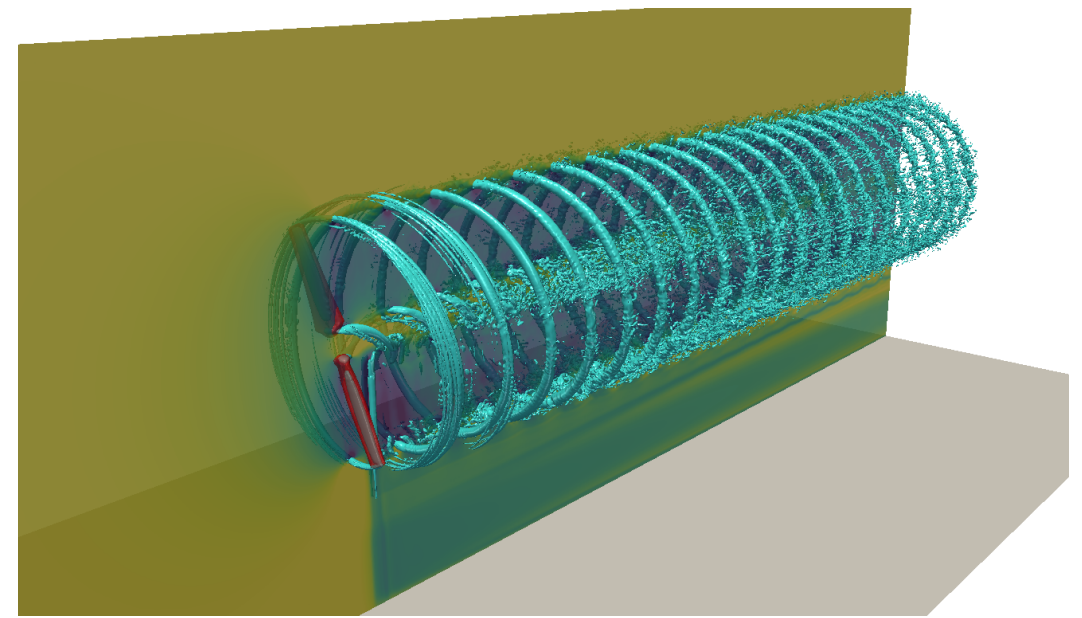

(b) AAL, $\epsilon_{c}=0.85 c, \epsilon_{t}=0.85 t$, integral velocity sampling

Figure 3. Contours of instantaneous streamwise velocity in a vertical plane passing through the rotor hub. The cyan isosurfaces are of the second invariant of the velocity-gradient tensor $Q$, and the red isosurfaces are of the body-force projection function $g$.

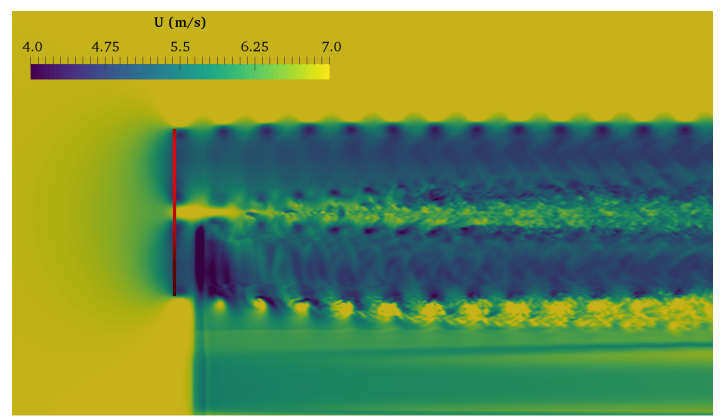

(a) $\mathrm{AL}, \epsilon=0.235 \mathrm{~m}$, point velocity sampling

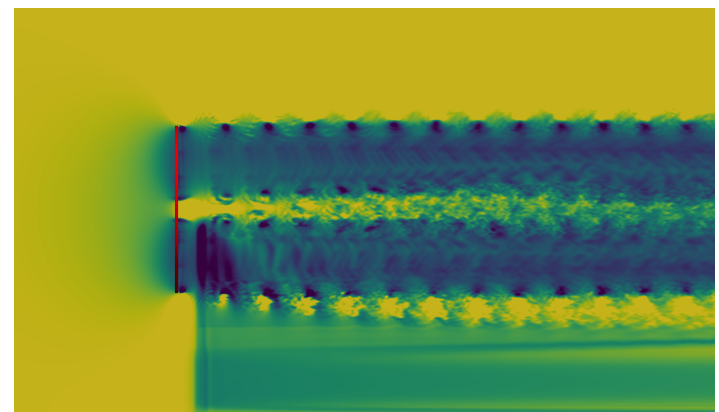

(b) AAL, $\epsilon_{c}=0.85 c, \epsilon_{t}=0.85 t$, integral velocity sampling

Figure 4. Contours of instantaneous streamwise velocity in a vertical plane passing through the rotor hub. The red line indicates the location of the rotor plane. 


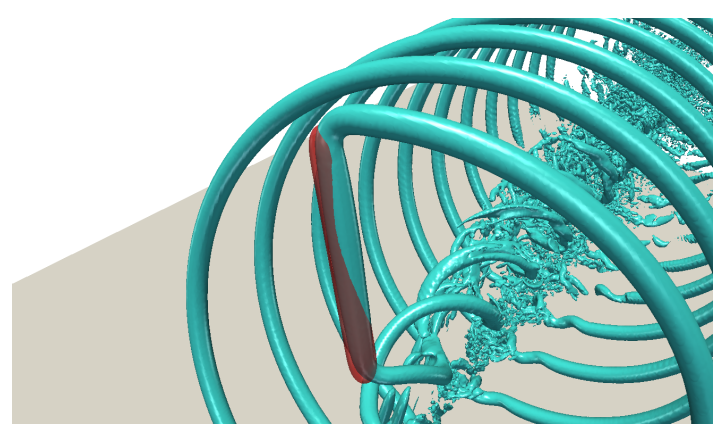

(a) AL, $\epsilon=0.235 \mathrm{~m}$, point velocity sampling

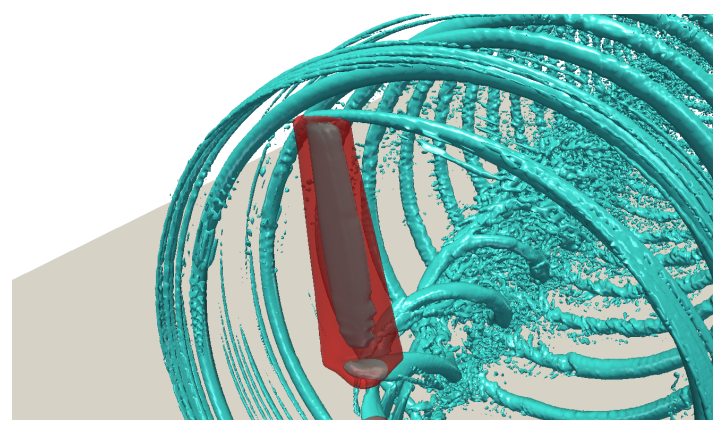

(b) AAL, $\epsilon_{c}=0.85 c, \epsilon_{t}=0.85 t$, integral velocity sampling

Figure 5. A close-up view of isosurfaces of the second invariant of the velocity-gradient tensor $Q$ (cyan) and isosurfaces of the body-force projection function $g$ (red).
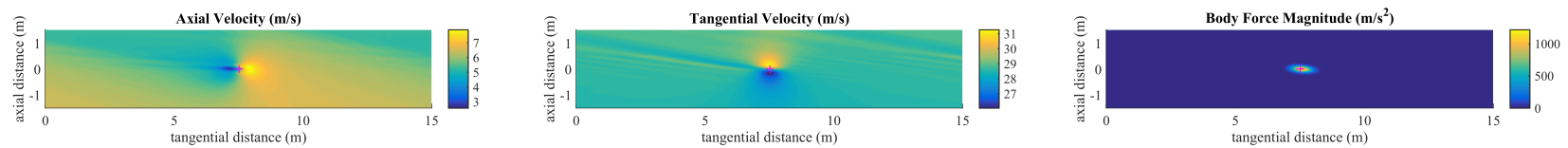

(a) AAL, $\epsilon_{c}=0.85 c, \epsilon_{t}=0.85 t$, integral velocity sampling
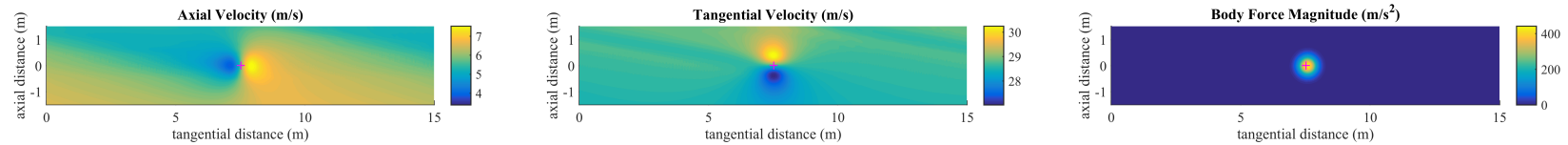

(b) AL, $\epsilon=0.235 \mathrm{~m}$, point velocity sampling
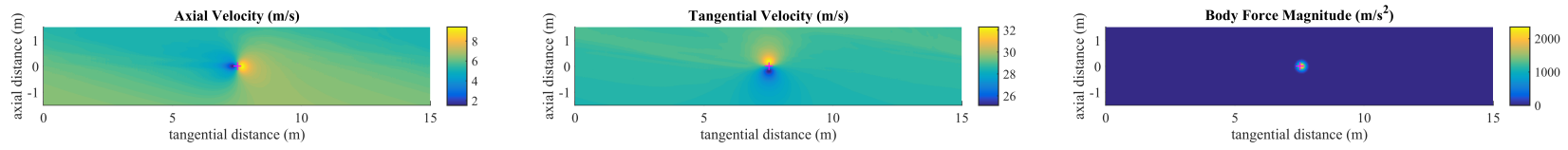

(c) AL, $\epsilon=0.15 \mathrm{~m}$, integral velocity sampling

Figure 6. The instantaneous axial velocity, tangential velocity, and body-force magnitude in a region sampled on an annulus at $75 \%$ of the blade radius. The annulus is unrolled and shown in a region around a single blade. The blade moves from left to right through the plot, and the axial flow is from bottom to top.

local streamlines, in effect cambering the body-force distribution. The axial velocity contours show similar differences between the AAL and AL methods. The main observation drawn from the two AL cases is that the flow field contours reflect the size of the body-force contours. In other words, the bound vortex around the actuator line scales with the body-force distribution size.

In Fig. 7, we sample the contours shown in Fig. 6 along tangential and axial lines that intersect the actuator line. These plots show the velocity distribution of the bound vortex. The AL case with the largest body-force projection width $(\epsilon=0.234 \mathrm{~m})$, creates the most diffuse bound vortex, which likely means the tip and root vortices near the blade will be similarly diffuse. The diffuse tip vortices cause incorrect downwash near the tip, overpredicting the tip loads, as we will show later. The AL with $\epsilon=0.15 \mathrm{~m}$ produces a less diffuse tip vortex, as expected, because the vorticity distribution follows the body-force distribution. At the part of the blade span, the AL with $\epsilon=0.15 \mathrm{~m}$ produces a less diffuse tip vortex than even the AAL case with $\epsilon_{c}=0.85 c=0.39 \mathrm{~m}$ and $\epsilon_{t}=0.85 t=0.11 \mathrm{~m}$, where $t$ is the blade thickness. At least in the chord direction, the AAL case body-force projection function is larger than in the $\mathrm{AL}(\epsilon=0.15 \mathrm{~m})$ case. In the thickness direction, the two cases have similar projection function width. Therefore, it makes sense that the AAL case bound vortex is more diffuse than the $\mathrm{AL}(\epsilon=0.15 \mathrm{~m})$ case.

Lift and drag forces can be applied as body forces in two ways: i) The body-force vector at all locations can point in the same direction as the original combined lift and drag vector, or ii) Eq. (3) can be used, 
which applies the body-force lift normal to streamlines. This can be seen in Fig. 8 which shows contours of instantaneous body-force magnitude on a plane perpendicular to an actuator line at $75 \%$ of the blade radius. On the plot are shown streamlines in white and body-force vector lines in red. The case that aligns the body forces using the simpler method i) is shown at left. The case that uses method ii) that employs Eq. (3) is shown at right. For method ii) the body-force lines are clearly perpendicular to the streamlines. For AAL cases with a thin $\epsilon_{t}$ value, method ii) projects force in a way similar to how pressure acts over the curved surface of a cambered airfoil.

Now that we have explored the actuator line flow field, we explore blade loading accuracy. In Fig. 9, we show the coefficients of blade-chord normal $\left(C_{n}\right)$ and tangential $\left(C_{t}\right)$ force along the blade span. These distributions are averaged over $6 \mathrm{~s}$ of simulation time and over both blades. Comparison is made to NREL Phase VI experimental measurement and blade-element-momentum theory calculations. The values are not well predicted inboard of $r / R=0.4$ to 0.5 . This inboard discrepancy may be caused by the lack of complex nacelle flow field effects or inadequate corrections to the airfoil lift/drag tables for three-dimensional, rotational flow effects. This part of the blade, though, does not create a significant portion of the blade loads and torque, so we are less concerned about the accuracy there as compared to outboard. Outboard, both $C_{n}$ and $C_{t}$ are slightly overpredicted. $C_{t}$ is predominantly responsible for the generation of torque and, hence,

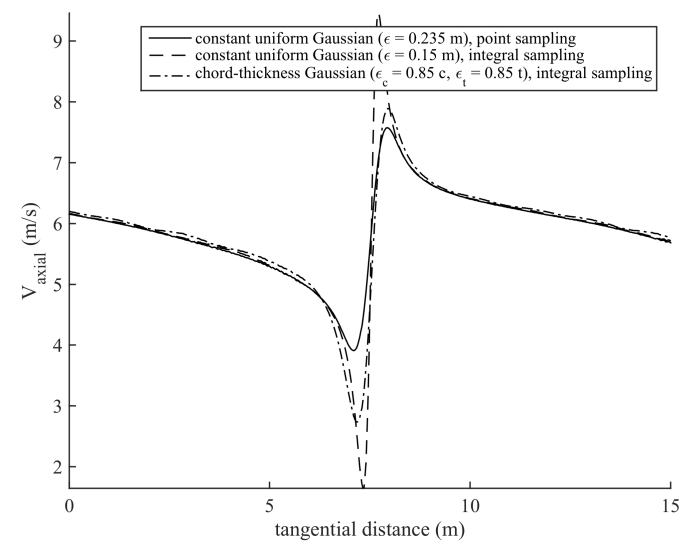

(a)

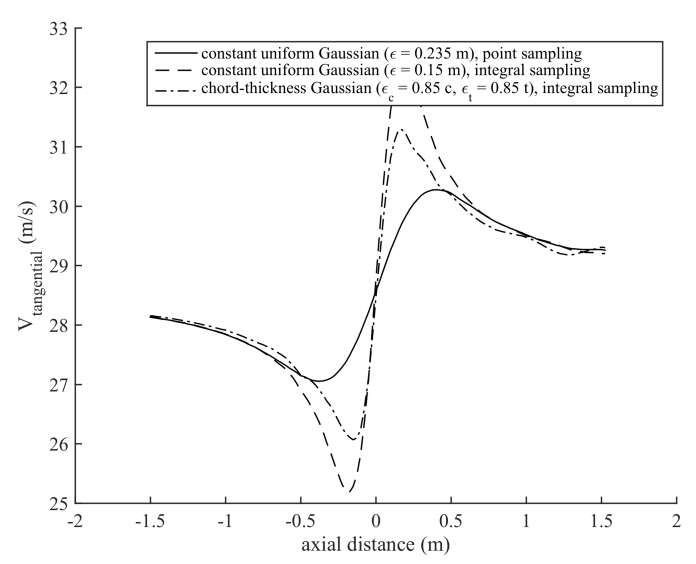

(b)

Figure 7. Profiles of the axial velocity on an axially oriented line (left) and of tangential velocity (in the blade-motion reference frame) on a tangentially oriented line, both passing through the actuator line at $75 \%$ of the blade span.

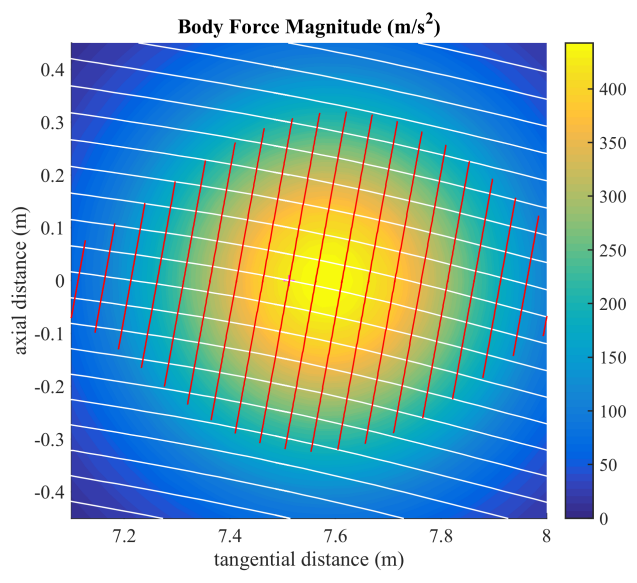

(a) AL, $\epsilon=0.235 \mathrm{~m}$, body-force alignment i)

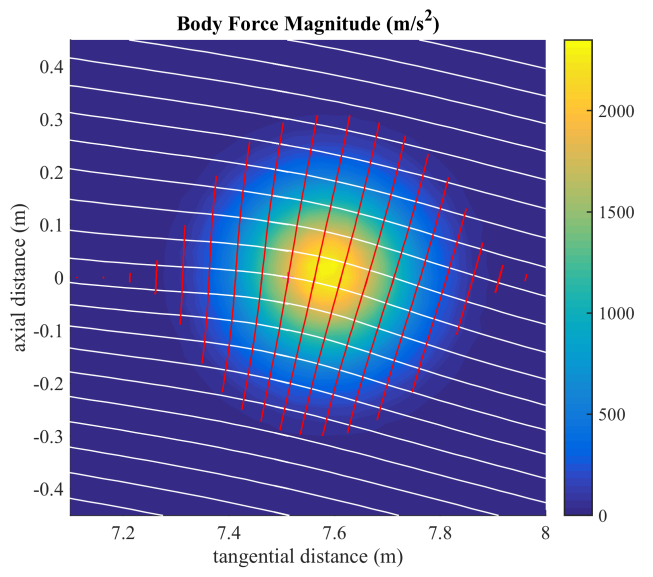

(b) AL, $\epsilon=0.15 \mathrm{~m}$, body-force alignment ii) (Eq. (3))

Figure 8. Contours of the instantaneous body-force magnitude in a region sampled on a plane perpendicular to the actuator line at $75 \%$ of the blade radius. Fluid streamlines are shown in white and body-force vector lines are shown in red. The blade moves from left to right through the plot, and the axial flow is from bottom to top. 


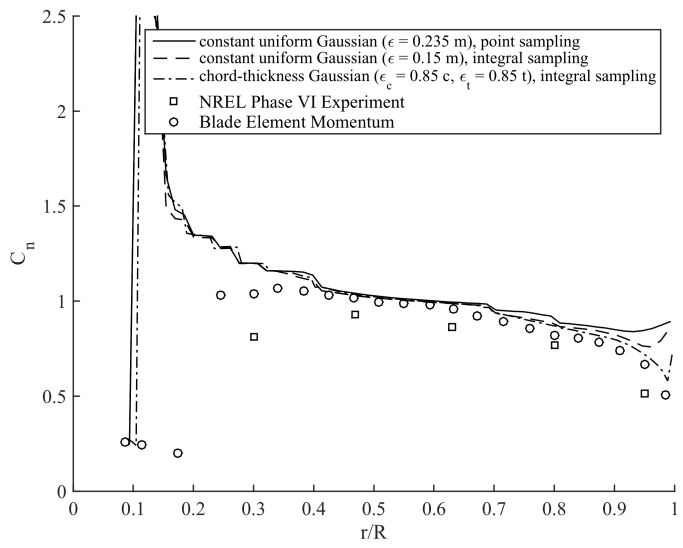

(a)

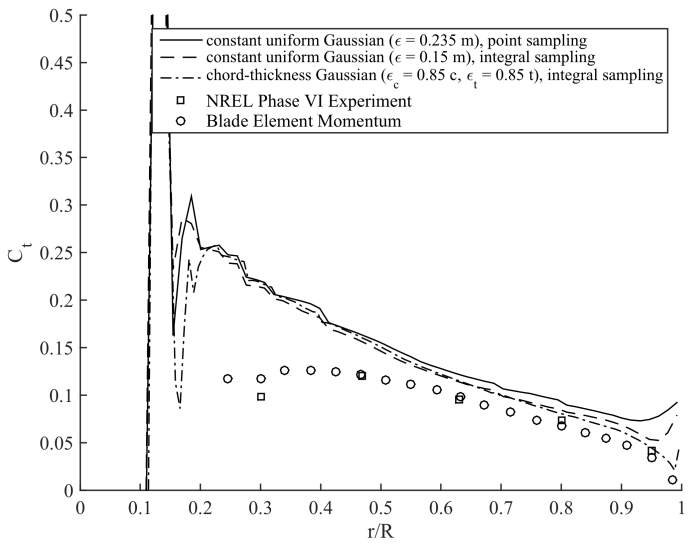

(b)

Figure 9. Coefficient of chord-line normal $\left(C_{n}\right)$ and chord-line tangential $\left(C_{t}\right)$ mean force along the blade span of the NREL Unsteady Aerodynamics Experiment Phase VI rotor from test sequence $H$.

power, so we expect power to be overpredicted, which is true and will be discussed later. Most notable, though, is the loading near the tip. For the AL cases, we observe an increase in $C_{n}$ and $C_{t}$ near the tip, but that effect is much less pronounced for the AAL case (only the last AL point exhibits an increase in value). This is likely caused by improper downwash caused by error in the tip vortex shape in the AL cases. Figure 3 clearly shows the AAL case to produce a tighter tip vortex than the AL $(\epsilon=0.15 \mathrm{~m})$ case.

The blade load distribution dictates the rotor aerodynamic power, which is shown in Fig. 10 for a 1.2-s period, enough time for the blade to pass through more than one full revolution. This is computed as the integrated blade torque multiplied by the rotor rotation rate. There are many features of interest in this plot. First, the time history of power for all cases has a periodic decrease every 0.42 s. This corresponds to each blade passing in front of the turbine tower, which we represent with an actuator body force, ${ }^{8}$ and its associated flow disruption. Next, there is significant scatter in the predicted power levels about the experimentally measured derived rotor power of $6 \mathrm{~kW}$. Admittedly, we ran multiple AL cases on the lower resolution mesh with point or integral velocity sampling and multiple AAL cases on the high-resolution mesh to find the value of $\epsilon$ that best matched the experimental power; in other words, predicted power is sensitive to the chosen value of $\epsilon$. That is why the low-resolution cases and the AAL case well match $6 \mathrm{~kW}$. Interestingly, though, with $\epsilon$ fixed, an increase in grid resolution creates an increase in power. This was also observed by Martínez-Tossas et al. ${ }^{5}$ Also, for the AL cases, integral versus point velocity sampling changes the value of $\epsilon$ required to well match the experimental rotor power. The reason for dependence of predicted power on body-force projection width, grid resolution, and velocity sampling method is unclear and must be explored further. Another interesting point drawn from this figure is that the power time histories from the point velocity sampling cases contains spurious high-frequency content. For actuator-line simulations with nonturbulent inflow, there is no reason this spurious content should be present. Our conjecture is that point sampling the velocity along the actuator line at the center of a discretely represented bound vortex using linear interpolation is prone to interpolation error. Looking back at Fig. 7, which shows profiles of velocity across the bound vortex centered upon the actuator line, we see that the velocity gradient here is steep, which would make point sampling via linear interpolation prone to error. This effect is exacerbated on the coarser mesh. In contrast, integral velocity sampling removes this spurious high-frequency content. This is to be expected as the integral method is an average over the entire bound vortex cross section and does not rely on interpolation. This is a promising finding that supports the use of the integral velocity sampling method, especially considering situations in which one would use actuator lines coupled with an aeroelastic tool to compute loads spectra; point velocity sampling may create spurious high-frequency load content. Because power is an integrated quantity, and the spurious content is present there, the spurious content is likely more pronounced at individual spanwise locations. 


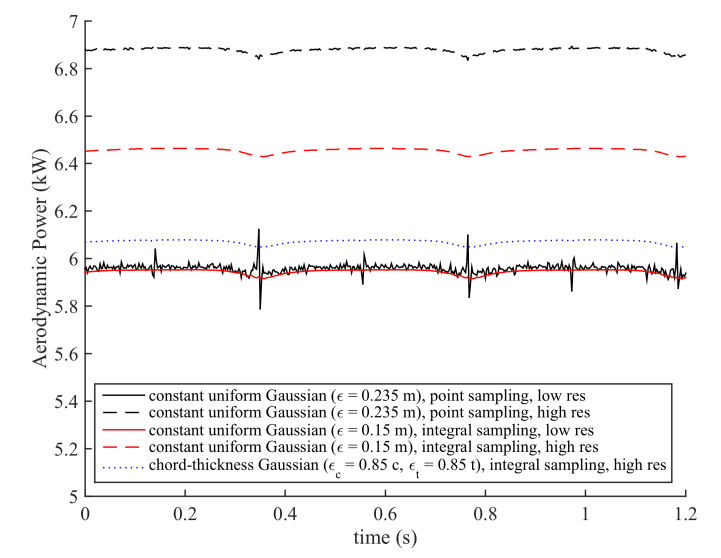

Figure 10. A time history of rotor aerodynamic power of one revolution of the simulated NREL Unsteady Aerodynamics Experiment Phase VI rotor from test sequence $H$.

\section{III.B. 5-MW Reference Turbine Case: Utility-Scale Wind Turbine}

This section presents the results of the utility-scale, NREL 5-MW reference turbine simulations. The mesh used for the computations is show in Fig. 11. It is similar to the mesh for the NREL Phase VI turbine cases. The mesh immediately within the rotor plane has cell lengths of $0.14 \mathrm{~m}$, which places roughly 10 grid cells across the chord length just before the tip cap. The mesh is also highly resolved where the tip and root vortices will convect downstream; however, this grid adaptation process was manual, and the tip vortices exit the fine mesh region due to wake expansion within one rotor diameter downstream. This is clearly visible in Fig. 11 (b) where the tip vortices are shown through isosurfaces of Q. The mesh is successively coarsened away from the rotor and wake to conserve mesh points. This mesh is roughly 90 million cells in size. The time step is $0.0025 \mathrm{~s}$, again to limit the tip motion to one grid cell per time step.

Two cases are shown: i) AL with $\epsilon=1.5 \mathrm{~m}$ and point velocity sampling, and ii) AAL with $\epsilon_{c}=0.4 c$ and $\epsilon_{t}=0.2 c$ and point velocity sampling. Figure 12 shows radial distributions of sampled axial velocity and computed lift per unit span along the actuator line for these four cases. The AAL case agrees well with blade-element-momentum theory. Although not shown here, we computed an AAL cases with $\epsilon_{t}=0.1 c$ with point and integral velocity sampling. The point-sampled case severely underpredicts the axial velocity and as a result the lift is underpredicted. We believe this happens because with $\epsilon_{t}=0.1 c$, the mesh only coarsely resolves the body force in the thickness direction in the outer part of the span where the blade chord becomes small. That means that the bound vorticity, which mirrors the body-force distribution, is coarsely resolved. Attempting to sample the center of a coarsely resolved bound vortex using linear interpolation is difficult, and may be the source of underprediction of velocity, yet to make any conclusive statement, we must explore the issue more deeply. Interestingly, the AAL case with this same narrow body-force distribution but with integral sampling behaves consistently with the shown AAL results and blade-element-momentum theory. This suggest that integral sampling is possibly a more robust way to sample the velocity along the actuator line. The standard AL case overpredicts axial velocity, near the tip. As in the Phase VI rotor case, this is likely because the relatively wide body-force distribution at the tip does not allow for as compact and strong a tip vortex, so the downwash is not as strong there as it should be.

Figure 13 show contours of instantaneous axial velocity and the second invariant of the velocity-gradient tensor $(\mathrm{Q})$ in a plane along the wake and through the rotor hub for the AL case and AAL case. It is clear that the AAL creates tighter, stronger tip and root vortices. This is especially apparent when looking at Q. The tip vortices rapidly lose strength with downstream distance only because the fine resolution sleeve of the mesh meant to capture these vortices does not expand with the wake, and the vortices exit that sleeve to coarser mesh that cannot well resolve them.

Figure 14 shows contours of axial body force in the rotor plane for the AL case i and AAL case iv. The AAL with its more compact force distribution in the blade thickness direction effectively compresses the body force into a smaller volume, so it is stronger in this case. 


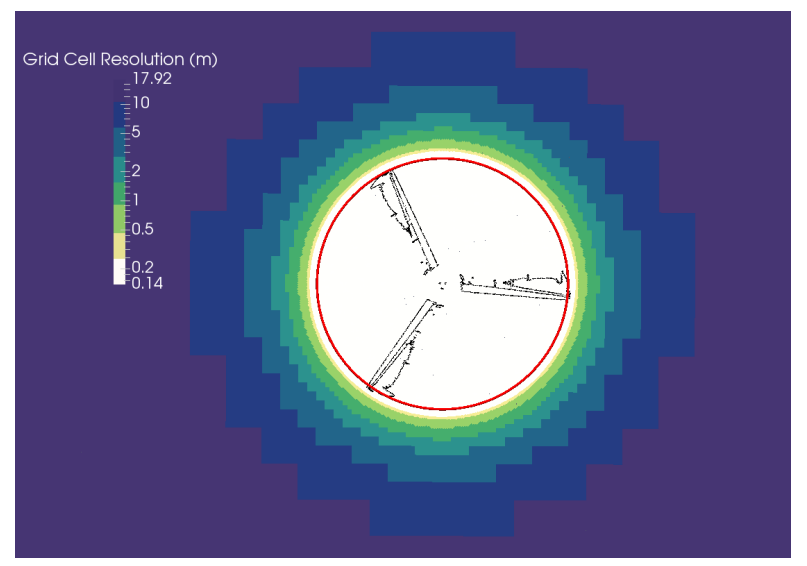

(a)

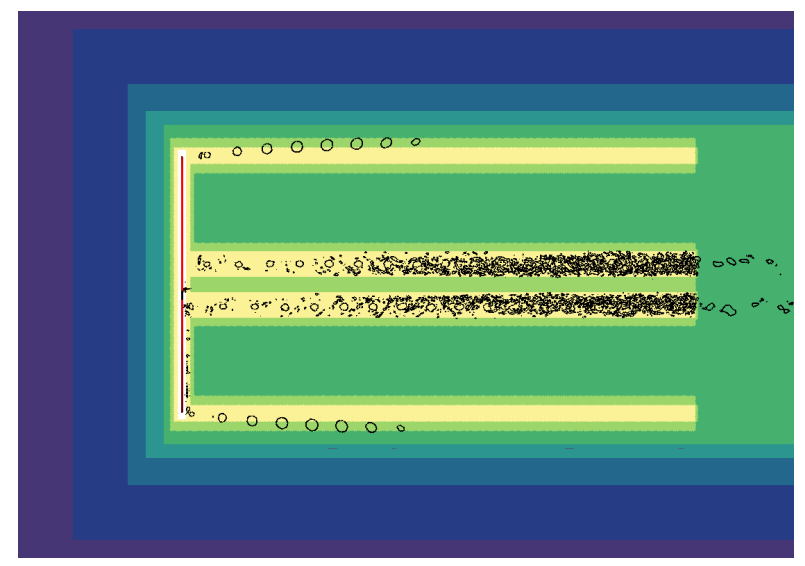

(b)

Figure 11. Cut planes through the mesh showing mesh resolution for the NREL 5-MW reference turbine simulation. View (a) is from the front and view (b) is perpendicular to the rotor plane through the rotor hub. The rotor plane is indicated in red. The black lines are contours of the second invariant of the velocity-gradient tensor $Q$.

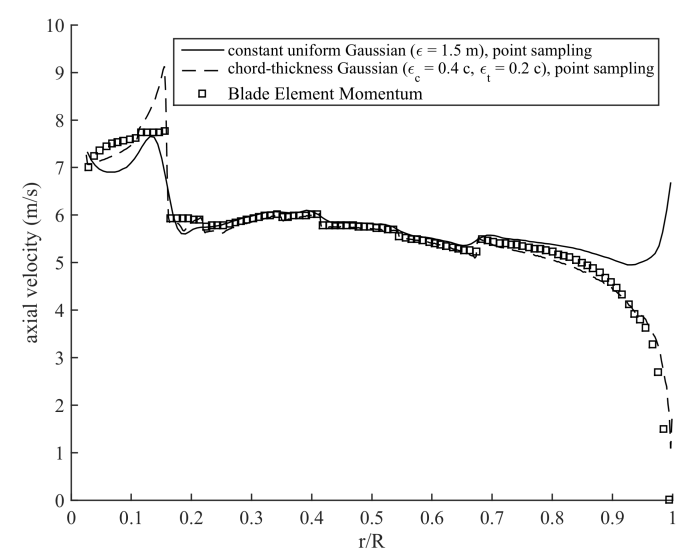

(a)

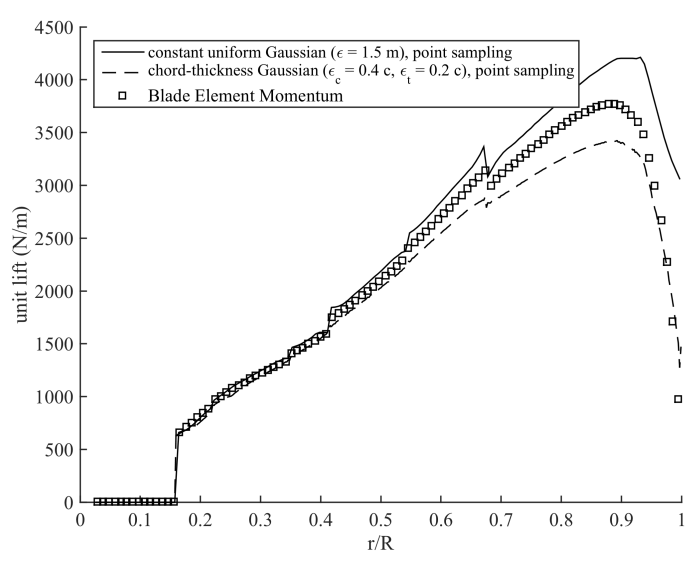

(b)

Figure 12. Sampled axial velocity (a) and lift per unit span (b) from the AL and AAL simulations of the NREL 5-MW reference turbine. 

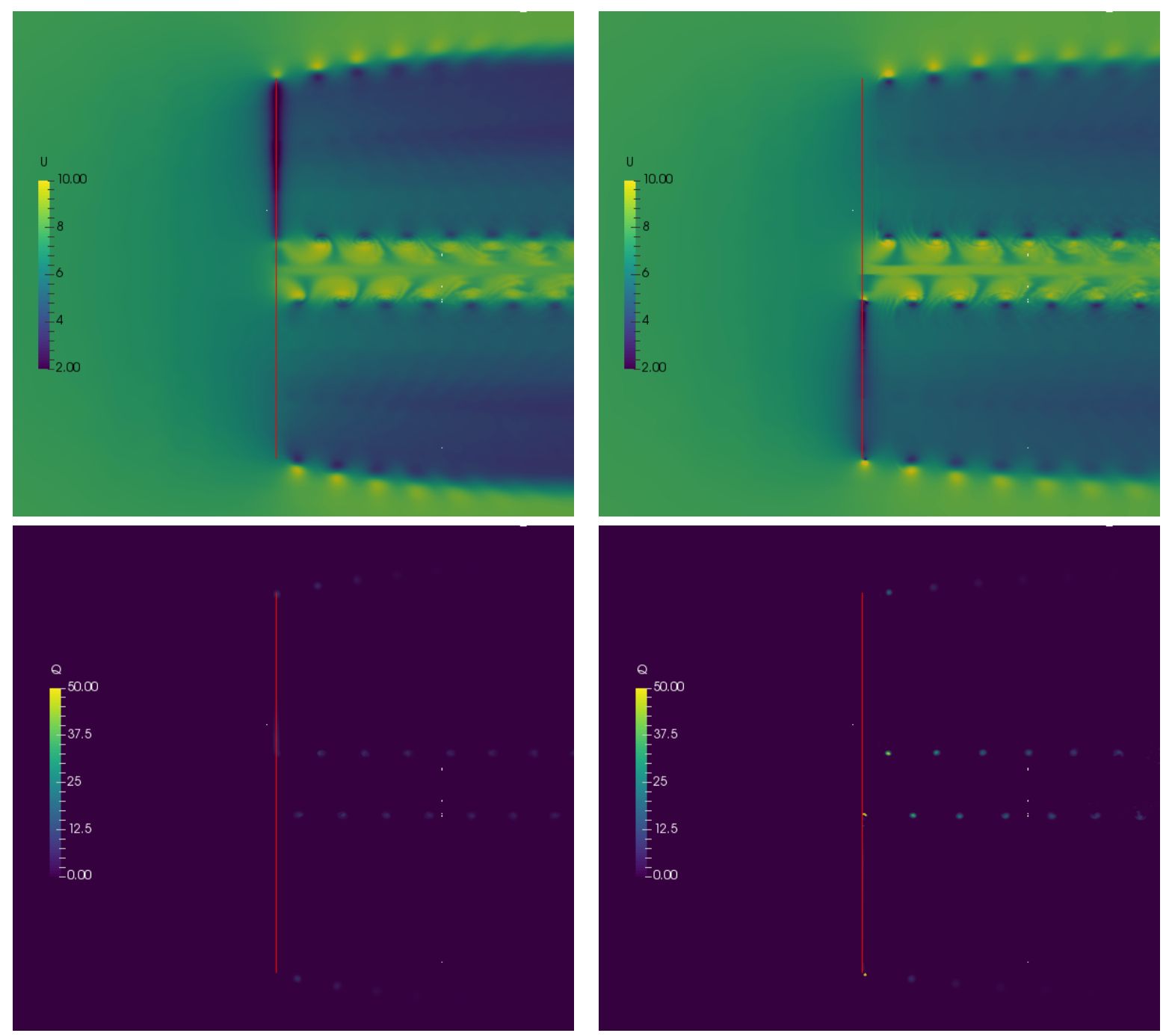

$\mathrm{AL}$

AAL

Figure 13. Contours in slices through the rotor hub showing the axial cross section of the wake flow from the NREL 5-MW reference turbine simulations. The left column is the standard actuator line and the right column is the advanced actuator line. The top row shows instantaneous axial velocity $(\mathrm{m} / \mathrm{s})$, and the bottom row shows the second invariant of the velocity-gradient tensor, $\mathrm{Q}(1 / \mathrm{s})$. The rotor position is denoted with a red line. 


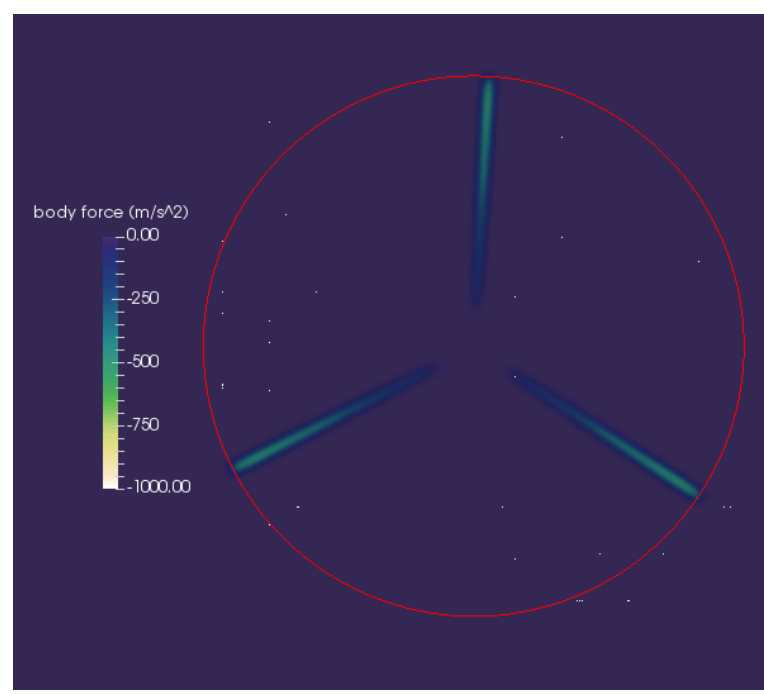

$\mathrm{AL}$

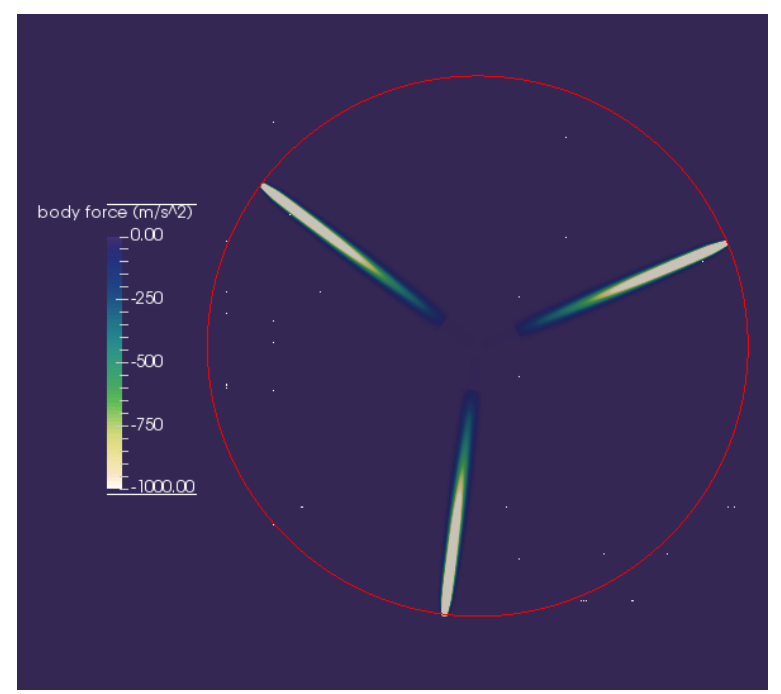

AAL

Figure 14. Contours in slices through the rotor plane showing the axial component of body force normalized by density and per unit volume computed in the NREL 5-MW reference turbine simulations. The left shows the contours for the standard actuator line and the right shows those of the advanced actuator line. The red circle denotes the rotor circle.

\section{Conclusion}

The actuator line method for representing wind turbine rotor aerodynamics when performing wind energy CFD has become prominent in the past few years. Most researchers use the actuator line method, or a close variant, proposed by Sørensen and Shen ${ }^{1}$ more than a decade ago. Although the method produces meaningful and useful results, it can be improved, which is the focus of this work. We outline a new blade/actuator line velocity sampling method that removes the ambiguity of sampling at points by performing a body-force projection-function-weighted velocity integral in space. We also explore a nonisotropic Gaussian body-force projection function that more realistically represents the distribution of force over a real blade. We test these new ideas on simulations of the NREL 5-MW reference utility-scale turbine, and the NREL Phase VI windtunnel test turbine with a 10-m rotor diameter. For both cases, we compare to blade-element-momentum theory calculations, and for the Phase VI rotor, we compare to experimental blade load distribution data.

One criticism of the standard actuator line method is that is does not capture the near wake details well, and the body-force distribution improvement presented here addresses that criticism. The nonisotropic Gaussian body-force projection function allows the advanced actuator line to have a fidelity level somewhere between standard actuator line and blade-resolved simulations. This improvement is not meant to produce blade boundary layer effects, but the more compact body-force distribution in the blade thickness direction allows for a more realistic and compact blade wake. The blade local flow field is also more representative of the flow about an airfoil. The more compact body-force distribution is also capable of better capturing the tip vortices and their resultant downwash and its effect on tip loads. This naturally alleviates the need for a blade tip correction, which is commonly used in actuator line calculations. We clearly show improvement in the actuator line simulations' agreement with the NREL Phase VI blade measured load distributions near the tip. Similarly, for the NREL 5-MW turbine case, the actuator line predictions of axial velocity and lift along the blade better agree with blade-element-momentum theory using the nonisotropic Gaussian. Furthermore, we believe the resultant wake flow fields are more accurate because the tip vortices are tighter than with the standard actuator line.

The integral freestream velocity sampling method is meant to both remove the ambiguity in where to sample the velocity and increase the robustness of the sampling. Our results show that integral sampling removes spurious high-frequency content that appears in the loads when we use point-sampled freestream velocity. Furthermore, with more tailored body-force projection functions than those used here, the location of point sampling becomes even more ambiguous, supporting the need for an integral sampling method. The estimated freestream velocity is sensitive to grid resolution and body-force projection function width. We believe this is not a sensitivity of the integral freestream velocity sampling method, but a sensitivity of the local freestream velocity to the size of the body-force distribution for the case of the wind turbine in which 
the lift vector is pointed predominantly into the oncoming wind.

Overall, the results are encouraging and these actuator line improvements show promise in creating a higher-fidelity, more accurate actuator line method without the need for empirical corrections. The increased fidelity comes at the cost of increased mesh resolution, but not at the cost and complexity level of bladeresolved simulations. The meshes used in this study are rather large only because high resolution is applied over the entire rotor disk. A much more efficient but slightly more complex meshing strategy could be used in which a cylinder of rotating mesh is placed within the rest of the mesh. This cylinder would rotate at the rotor speed and would only have high resolution in regions concentrated about the actuator lines, greatly reducing the mesh cell count.

In the near future, we would like to make comparisons of computed to measured wakes and tip vortices. To do this, we plan to simulate the MEXICO rotor, for which there are particle image velocimetery measurements of the tip vortices near the rotor. We also show the outstanding issues of strong dependence of predicted loads and power to mesh resolution and body-force distribution size, which requires more attention. It could also be beneficial to perform more testing on a stationary, nonrotating wing, possibly comparing with high-quality geometry-resolved computations.

\section{Acknowledgments}

This work was supported by the U.S. Department of Energy (DOE) under Contract No. DE-AC3608GO28308 with the National Renewable Energy Laboratory. Funding for the work was provided by the DOE Office of Energy Efficiency and Renewable Energy, Wind Energy Technologies Office. L.A.M.T. and C.M. acknowledge support from the National Science Foundation (OISE 1243482). Computations were performed on the National Renewable Energy Laboratory's Peregrine high-performance computing system. The U.S. Government retains and the publisher, by accepting the article for publication, acknowledges that the U.S. Government retains a nonexclusive, paid-up, irrevocable, worldwide license to publish or reproduce the published form of this work, or allow others to do so, for U.S. Government purposes.

\section{References}

${ }^{1}$ Sørensen, J. N. and Shen, W. Z., "Numerical Modeling of Wind Turbine Wakes," Journal of Fluids Engineering, Vol. 124, 2002, pp. 393-399.

${ }^{2}$ Mikkelsen, R., Sørensen, J. N., Øye, S., and Troldborg, N., "Analysis of Power Enhancement for a Row of Wind Turbines Using the Actuator Line Technique," Journal of Physics: Conference Series - The Science of Making Torque from Wind, Vol. 75, No. 012044, 2007.

${ }^{3}$ Troldborg, N., Actuator Line Modeling of Wind Turbine Wakes, Ph.D. thesis, Technical University of Denmark, Lyngby, Denmark, 2008.

${ }^{4}$ Troldborg, N., Zahle, F., Réthoreé, P.-E., and Sørensen, N. N., "Comparison of the Wake of Different Types of Wind Turbine CFD Models," 50th AIAA Aerospace Sciences Meeting including the New Horizons Forum and Aerospace Exposition, Nashville, TN, Jan. 9-12, 2012, AIAA, Washington D.C., 2012, AIAA Paper 2012-0237.

${ }^{5}$ Martínez-Tossas, L. A., Churchfield, M. J., and Leonardi, S., "Large Eddy Simulations of the Flow Past Wind Turbines: Actuator Line and Disk Modeling," Wind Energy, Vol. 18, 2015, pp. 1047-1060.

${ }^{6}$ Churchfield, M. J., Lee, S., Michalakes, J., and Moriarty, P. J., "A Numerical Study of the Effects of Atmospheric and Wake Turbulence on Wind Turbine Dynamics," Journal of Turbulence, Vol. 13, No. 14, 2012.

${ }^{7}$ Churchfield, M. J., Lee, S., Moriarty, P. J., Martínez, L. A., Leonardi, S., Vijayakumar, G., and Brasseur, J. G., "A Large-Eddy Simulation of Wind-Plant Aerodynamics," 50th AIAA Aerospace Sciences Meeting including the New Horizons Forum and Aerospace Exposition, Nashville, TN, Jan. 9-12, 2012, AIAA, Washington D.C., 2012, AIAA Paper $2012-537$.

${ }^{8}$ Churchfield, M. J., Lee, S., Moriarty, P. J., Hao, Y., Lackner, M. A., Barthelmie, R., Lundquist, J. K., and Oxley, G., "A Comparison of the Dynamic Wake Meandering Model, Large-Eddy Simulation, and Field Data at the Egmond aan Zee Offshore Wind Plant," 53rd AIAA Aerospace Sciences Meeting, Kissimmee, FL, Jan. 5-9, 2015, AIAA, Washington D.C., 2015, AIAA Paper 2015-0724.

${ }^{9}$ Churchfield, M. J., Li, Y., and Moriarty, P. J., "A Large-Eddy Simulation Study of Wake Propagation and Power Production in an Array of Tidal-Current Turbines," Philosophical Transactions of the Royal Society A, Vol. 371, 2013, pp. 20120421.

${ }^{10} \mathrm{Lu}, \mathrm{H}$. and Porté-Agel, F., "Large-Eddy Simulation of A Very Large Wind Farm in an Stable Atmospheric Boundary Layer," Physics of Fluids, Vol. 23, 2011, pp. 065101.

${ }^{11}$ Bhaganagar, K. and Debnath, M., "Implications of Stably Stratified Atmospheric Boundary Layer Turbulence on the Near-Wake Structure of Wind Turbines," Energies, Vol. 7, 2014, pp. 5740-5763.

12 Jha, P. K., Churchfield, M. J., Moriarty, P. J., and Schmitz, S., "Guidelines for Volume Force Distributions Within Actuator Line Modeling of Wind Turbines on Large-Eddy Simulation-Type Grids," Journal of Solar Energy Engineering, Vol. 136, 2014.

${ }^{13}$ Shives, M. and Crawford, C., "Mesh and Load Distribution Requirements for Actuator Line CFD Simulations," Wind Energy, Vol. 16, No. 8, 2012, pp. 1183-1196. 
${ }^{14}$ Fleming, P. A., Gebraad, P. M. O., Lee, S., van Wingerden, J.-W., Johnson, K., Churchfield, M., Michalakes, J., Spalart, P., and Moriarty, P., "Evaluating Techniques for Redirecting Turbine Wakes using SOWFA," Renewable Energy, Vol. 70, 2014, pp. 211-218.

${ }^{15}$ Fleming, P. A., Gebraad, P. M. O., Lee, S., van Wingerden, J.-W., Johnson, K., Churchfield, M., Michalakes, J., Spalart, P., and Moriarty, P., "Simulation Comparison of Wake Mitigation Control Strategies for a Two-Turbine Case," Wind Energy, Vol. 18, 2015, pp. 2135-2143.

${ }^{16}$ Forsythe, J. R., Lynch, E., Polsky, S., and Spalart, P., "Coupled Flight Simulator and CFD Calculations of Ship Airwake using HPCMP CREATE-AV Kestrel," 53rd AIAA Aerospace Sciences Meeting, Kissimmee, FL, Jan. 5-9, 2015, AIAA, Washington D.C., 2015, AIAA Paper 2015-0556.

${ }^{17}$ Jones, M. and Paterson, E. G., "Evolution of the Propeller Near-Wake and Potential Energy in a Thermally-Stratified Environment," Oceans 16, September 19-23, Monterey, California, 2016.

${ }^{18}$ Martínez-Tossas, L. A., Churchfield, M. J., and Meneveau, C., "Optimal Smoothing Length Scale for Actuator Line Models of Wind Turbine Blades," Wind Energy, 2017, Accepted, in press.

${ }^{19}$ Mittal, A., Sreenivas, K., Taylor, L., and Hereth, L., "Improvements to the Actuator Line Modeling for Wind Turbines," 53rd AIAA Aerospace Sciences Meeting, Kissimmee, FL, Jan. 5-9, 2015, AIAA, Washington D.C., 2015, AIAA Paper 20150216.

${ }^{20}$ Shen, W.-Z., Sørensen, J.-N., and Mikkelsen, R., "Tip Loss Correction for Actuator/Navier-Stokes Computations," Journal of Solar Energy Engineering, Vol. 127, 2005, pp. 209-213.

${ }^{21}$ OpenCFD Ltd. (ESI Group), "OPENFOAM - The Open Source CFD Toolbox," available online at http://www.openfoam.com, accessed June 2, 2015.

${ }^{22}$ Issa, R. I., "Solution of the Implicitly Discretized Fluid Flow Equations by Operator-Splitting," Journal of Computational Physics, Vol. 62, 1985, pp. 40-65.

${ }^{23}$ Issa, R. I., Gosman, A. D., and Watkins, A. P., "The Computation of Compressible and Incompressible Recirculating Flow by a Non-iterative Implicit Scheme," Journal of Computational Physics, Vol. 62, 1986, pp. 66-82.

${ }^{24}$ Rhie, C. M. and Chow, W. L., "Numerical Study of the Turbulent Flow Past an Airfoil with Trailing Edge Separation," AIAA Journal, Vol. 21, No. 11, 1983, pp. 1525-1532.

${ }^{25}$ Hand, M. M., Simms, D. A., Fingersh, L. J., Jager, D. W., Cotrell, J. R., Schreck, S., and Larwood, S. M., "Unsteady Aerodynamics Experiment Phase VI: Wind Tunnel Configurations and Available Data Campaigns," Tech. Rep. NREL/TP500-29955, National Renewable Energy Laboratory, Golden, Colorado, 2001.

${ }^{26}$ Shen, W.-Z., Hansen, M. O. L., and Sørensen, J. N., "Determination of the Angle of Attack on Rotor Blades," Wind Energy, Vol. 12, No. 91-98, 2009.

${ }^{27}$ Spalart, P. R., "Body-Force Representation of Airfoils," personal communication with M. Churchfield, May, 2011.

${ }^{28}$ Jonkman, J., Butterfield, S., Musial, W., and Scott, G., "Definition of a 5-MW Reference Wind Turbine for Offshore System Deployment," Tech. Rep. NREL/TP-500-38060, National Renewable Energy Laboratory, 2009. 